\title{
Classifications on the travelling wave solutions to the (3+1)-dimensional generalized KP and Jimbo-Miwa equations
}

\author{
Ozlem Isik $^{1, *}$, Ozge Irmak Degirmenci ${ }^{2}$, and Hasan Bulut ${ }^{2}$ \\ ${ }^{1}$ Kirklareli University, Department of Mathematics, 39100 Kirklareli, Turkey \\ ${ }^{2}$ Firat University, Department of Mathematics, 23119 Elazig, Turkey
}

\begin{abstract}
In this paper, we use the powerful $\tan (F(\xi) / 2)$-expansion method with the help of Wolfram Mathematica 9 to investigate the solution structures of two well-known nonlinear evolution equations, namely; the (3+1)-dimensional generalized KP and the (3+1)-dimensional Jimbo-Miwa equations. We obtain new solutions such as hyperbolic function, trigonometric function, exponential function and rational function solutions. We plot two- and three-dimensional graphics of some obtained results using the same program, Wolfram Mathematica 9.
\end{abstract}

\section{Introduction}

Many phenomena in nonlinear science can be expressed in the form of nonlinear partial differential equations. In recent years, various methods have been used to find the solitary wave solutions to such class of problems such as the sine-cosine function method [1], the variational iteration method [2], the Homotopy perturbation method [3], the exp-function method [4], the generalized tanh-coth method [5], the extended hyperbolic function method [6], the modified simple equation method [7], the sine-Gordon expansion method [8] and so on. However, in this study, we employ the effective $\tan (F(\xi) / 2)$-expansion method to investigate the new solutions of the (3+1)-dimensional generalized Kadomtsev-Petviashvili (KP) equation [9] and the (3+1)-dimensional Jimbo-Miwa equation [10]. The KP equation is introduced by [11] to describe the model of nonlinear wave motion that arises in the field of mathematical physics. The Jimbo-Miwa equation is used to describe $(3+1)$-dimensional wave model in the field of physics [12]. Various methods have been implemented to obtain different types of solutions to the equations mentioned above such as Backlund transformation [13], the simplified Hirota's method [14], the simplified Hirota's direct method [15], the exp-function method [4], the generalized tanh method [16], the Xu's stable-range method and logarithmic generalization of stable-range method [17], the Kudryashov method [18], the homogeneous balance method [19] and many more methods. Moreover fractional concepts are investigated in [20, 21, 22].

* Corresponding author: ozlem.isik@klu.edu.tr 


\section{The $\tan (F(\xi) / 2)$-expansion method}

In this section, we explain the $\tan (F(\xi) / 2)$-expansion method and how it can be applied to obtain the solutions of a given nonlinear PDE as in the following

$$
P\left(u, u^{2}, u_{x}, u_{x x}, u_{t}, u_{t t}, u_{x t}, \ldots\right)=0 .
$$

Performing the travelling wave transformation $u(x, t)=U(\xi), \xi=x-k t$, Eq. (2.1) reduces to the following ODE

$$
Q\left(U^{\prime}, U^{\prime \prime}, U^{\prime \prime \prime}, \ldots\right)=0
$$

The solution of Eq. (2.2) is given as follows

$$
U(\xi)=\sum_{i=0}^{m} A_{i}\left(p+\tan \left(\frac{F(\xi)}{2}\right)\right)^{i}+\sum_{i=1}^{m} B_{i}\left(p+\tan \left(\frac{F(\xi)}{2}\right)\right)^{-i}, A_{m} \neq 0, B_{m} \neq 0,
$$

where $m \in \mathbb{Z}^{+}$that can be determined by balancing the highest order derivative and the highest nonlinear term in Eq. (2.2). The coefficients $A_{i}, B_{i},(0 \leq i \leq m)$ are constants to be determined and $F=F(\xi)$ satisfies the nonlinear ODE [23]:

$$
F^{\prime}(\xi)=a \sin (F(\xi))+b \cos (F(\xi))+c .
$$

Substituting Eq. (2.3) into Eq. (2.2) produces a system of algebraic equations for $\tan (F(\xi) / 2)^{i}, \cot (F(\xi) / 2)^{i}$. After collecting this separated system of equations, we calculate $k, p, A_{0}, A_{1}, B_{1}, \ldots, A_{m}, B_{m}$. For solutions of Eq. (2.4), see [23].

\section{Applications}

In this section, implementation of the $\tan (F(\xi) / 2)$-expansion method is given.

1. Let us consider the $(3+1)$-dimensional generalized KP equation given by [9]:

$$
u_{x x x y}+3\left(u_{x} u_{y}\right)_{x}+u_{t x}+u_{t y}-u_{z z}=0 \text {, }
$$

performing the transformation $u(x, \mathrm{y}, \mathrm{z}, t)=U(\xi), \xi=x+y+\beta z-k t$, (3.1) becomes:

$$
U^{\prime \prime \prime}+3\left(U^{\prime}\right)^{2}-\left(2 k+\beta^{2}\right) U^{\prime}=0 \text {. }
$$

Using the balancing principle on Eq. (3.2) by considering the terms $\left(U^{\prime}\right)^{2}$ and $U^{\prime \prime \prime}$, we obtain $m=1$. Together with Eq. (2.3), we consider the solution of Eq. (3.1) to be:

$$
U(\xi)=A_{0}+A_{1}\left(p+\tan \left(\frac{F(\xi)}{2}\right)\right)+B_{1}\left(p+\tan \left(\frac{F(\xi)}{2}\right)\right)^{-1} .
$$

Substituting Eq. (3.3) into Eq. (3.2) and simplifying together with Eq. (2.4), we obtain a system of algebraic equations in $A_{1}, B_{1}, p, k, a, b, c$ and solving this system we obtain families of solutions in each case by considering the solutions of Eq. (2.4) that can be found in [23]. One of the solutions of Eq. (3.1) for Case-1 is given below.

Case1: $A_{1}=0, B_{1}=b+c-2 a p-b p^{2}+c p^{2}, k=\left(a^{2}+b^{2}-c^{2}+\beta^{2}\right) / 2$

Solution4: when $a^{2}+b^{2}-c^{2}<0, c \neq 0$ and $b=0$ 


$$
u_{1,4}(x, y, z, t)=c\left(c-2 a p+c p^{2}\right) /\left[c p-a^{2}+\sqrt{c^{2}-a^{2}} \tan \left(\omega_{1,4}(x, y, z, t)\right)\right],
$$

where $\omega_{1,4}(x, y, z, t)=\sqrt{c^{2}-a^{2}}\left(2(x+y+\beta z)+\left(\beta^{2}+c^{2}-a^{2}\right) t\right) / 4$.

2. Consider the (3+1)-dimensional Jimbo-Miwa equation [10]:

$$
u_{x x x y}+3 u_{y} u_{x x}+3 u_{x} u_{x y}+2 u_{y t}-3 u_{x z}=0
$$

performing the transformation $u(x, \mathrm{y}, \mathrm{z}, t)=U(\xi), \xi=x+y+\beta z-k t$, Eq. (3.5) becomes

$$
U^{\prime \prime \prime}+3\left(U^{\prime}\right)^{2}-(2 k+3 \beta) U^{\prime}=0 .
$$

Using the balancing principle on Eq. (3.6) by considering the terms $\left(U^{\prime}\right)^{2}$ and $U^{\prime \prime \prime}$, we obtain $m=1$. Together with Eq. (2.3), we consider the solution of Eq. (3.5) to be:

$$
U(\xi)=A_{0}+A_{1}\left(p+\tan \left(\frac{F(\xi)}{2}\right)\right)+B_{1}\left(p+\tan \left(\frac{F(\xi)}{2}\right)\right)^{-1} .
$$

Substituting Eq. (3.7) into Eq. (3.6) and simplifying together with Eq. (2.4), we obtain a system of algebraic equations in $A_{1}, B_{1}, p, k, a, b, c$. We solve this system and consequently families of solutions to Eq. (3.5) is obtained by considering the solutions of Eq. (2.4) that can be found in [23]. A family of solution to (3.5) is given for the Case1 as follows

\section{Case1}

$$
A_{1}=b-c, B_{1}=-a^{2}-b^{2}-3 c A_{1}+3 b c-2 c^{2}+2 k+3 \beta /\left(3 A_{1}\right), a=-\sqrt{-b^{2}+c^{2}+2 k+3 \beta}
$$

Solution-9: when $b=0$ and $a=c$

$$
u_{9}(x, y, z, t)=c(1-p)+4 /[2(x+y+\beta z)+3 \beta t] .
$$

\section{Graphics Plot}

In this section we present two- and three-dimensional graphics of some obtained solutions.
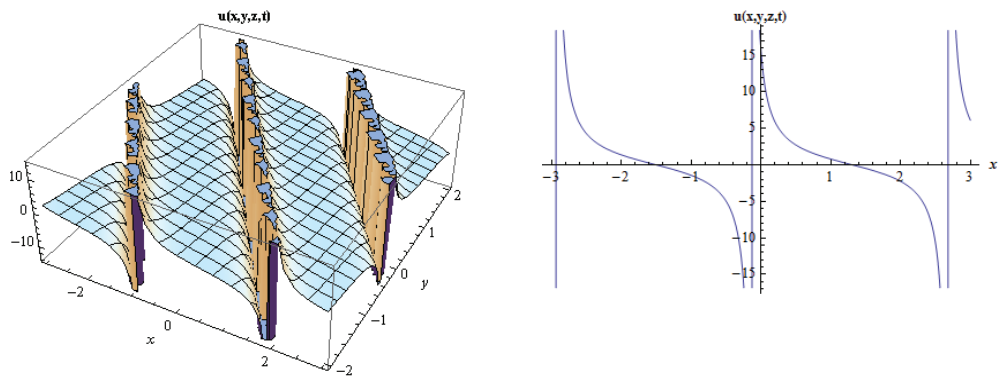

Fig. 1. 3D and 2D surfaces of Eq. (3.4) by considering the values $\beta=0.5, a=2, c=3, p=0.7, t=0.02$, $z=0.01,-3<x<3,-2<y<2$ and $y=0.03$ for the $2 \mathrm{D}$ graphic. 

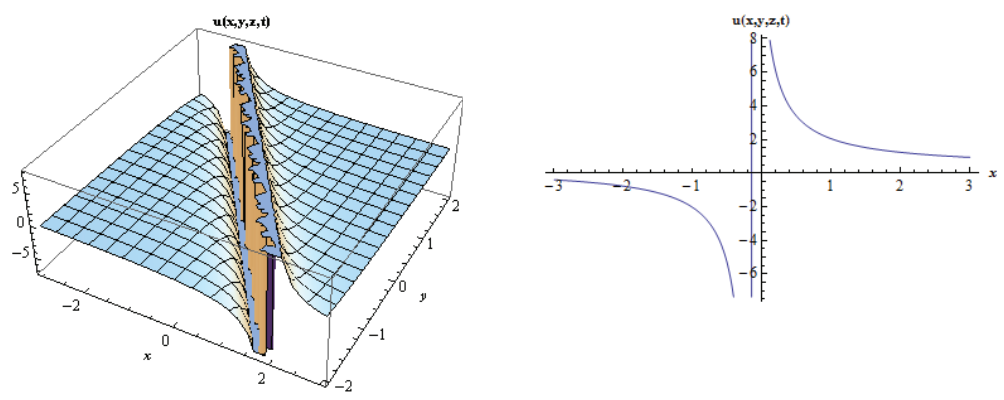

Fig. 2. $3 \mathrm{D}$ and $2 \mathrm{D}$ surfaces of Eq. (3.8) by considering the values $\beta=3, c=1, p=0.7, t=0.02, z=0.01$, $-3<x<3,-2<y<2$ and $y=0.03$ for the $2 \mathrm{D}$ graphic.

\section{Conclusions}

In this manuscript, we employed the $\tan (F(\xi) / 2)$-expansion method to $(3+1)$-dimensional generalized KP and Jimbo-Miwa equations. We obtained various types of solutions as, trigonometric, hyperbolic, exponential and rational functions. We plot two- and threedimensional graphics of some obtained solutions by using Wolfram Mathematica9. These solutions are tested and it is verified that they satisfy their corresponding equations. We compared our results with the results obtained in [9] and [10] and we observe that our results are new with different solution structures.

\section{References}

1. A.J.M. Jawad, IJRRAS, 13, 1 (2012)

2. A.M. Wazwaz, Computers and Mathematics with Applications, 54, (2007)

3. T.A. Nofel, Applied Mathematitcs, 5, (2014)

4. T. Ozis, I. Aslan, Phcysics Letters A, 372 (2008)

5. G.G. Hernan, Advances in Difference Equations, 9, 138 (2015)

6. Y. Shang, Chaos, Solutions and Fractals, 36, 3 (2008)

7. M. Mirzazadeh, Information Sciences Letters, 3, 1 (2014)

8. H. Bulut, T.A. Sulaiman, H.M. Baskonus, Opt. Quant. Electron., 48, 564 (2016)

9. W. Ma, Z. Zhu, Applied Mathematics and Computation, 218 (2012)

10. W. Ma, J. Lee, Chaos, Solitons and Fractals, 42 (2009)

11. B.B. Kadomtsev, V.I. Petviashvili, Sov. Phys. Dokl., 15 (1970)

12. M.T. Darvishi, M. Najafi, World Academy of Science, Engineering and Technology, 55, (2011)

13. W. Ma, A. Abdeljabbar, Applied Mathematics Letters, 25, 7 (2012)

14. A.M. Wazwaz, Commun. Nonlinear Sci. Numer. Simulat., 17 (2012)

15. A.M. Wazwaz, S.A. El-Tantawy, Nonlinear Dyn., 84 (2016)

16. W. Hong, K. Oh, Computers and Mathematics with Applications, 39 (2000)

17. B. Cao, Acta. Appl. Math., 112, 2 (2010)

18. N. Kadkhoda, CJMS, 4, 2 (2015)

19. M. Eslami, CJMS, 2, 2 (2014)

20. E. Bas, F. Metin, Advances in Difference Equations, 2013 (2013)

21. E. Bas, ActaScientiarum. Technology, 37.2 (2015)

22. E. Bas, R.Yilmazer, E. Panakhov, The Scientific World Journal, 2013 (2013)

23. J. Manafian, Optik, 127 (2016) 